\title{
LAS REDES SOCIALES Y EL MARKETING DE STAKEHOLDERS EN LA GESTIÓN DE LOS CANALES DE DISTRIBUCIÓN DE PRODUCTOS TURÍSTICOS: EL CASO DE BOOKINGFAX
}

\author{
Prof. Dr. Ramón Cuadrado Marqués ${ }^{1}$ \\ Departamento de Marketing y Comunicación. Universidad de Cádiz \\ ramon.cuadrado@uca.es
}

(Fecha envío: 30/10/15 - Fecha aceptación: 09/12/15)

\begin{abstract}
Resumen
El cambio experimentado en el comportamiento del consumidor turístico ha llevado a un mayor uso de las redes sociales como fuente de información en el proceso de decisión de compra. Además, las empresas del sector estiman que estas herramientas constituyen en la actualidad un elemento clave en el proceso de comunicación con el cliente. Sin embargo, sólo aquellas que consiguen la confianza del consumidor logran acceder al cliente, dificultándose y encareciéndose el coste por contacto. Si bien, se considera que la gestión de las relaciones con los distintos agentes participantes en el canal de distribución contribuye a mejorar el acceso de las empresas turísticas a su público objetivo, una necesidad que se ve intensificada por la intangibilidad del producto ofertado y la consiguiente exigencia de confianza por parte del cliente.
\end{abstract}

Bajo el enfoque del marketing de stakeholders se propone la gestión de las relaciones con todos los agentes presentes en el canal, constituyendo este enfoque una herramienta especialmente útil en la gestión de relaciones de interés y confianza entre ambos. Para analizar este fenómeno e ilustrar la utilidad del enfoque propuesto, bajo la metodología de estudio de casos, centraremos la investigación en el modelo de negocio de la empresa bookingfax, la cual emplea su capital relacional como base del mismo.

\section{Palabras clave}

cadena de valor, marketing, stakeholder, turoperador, relaciones, agencias de viajes

\begin{abstract}
The shift in touristic consumer behaviour has led to a more intense use of social media as a source of information in the buying decision-making process. Furthermore, businesses in this sector consider that these tools are nowadays a key element in the process of communicating with the customer. Nevertheless, only those companies that gain consumers' trust can access them, complicating and raising the cost by impact. Even though, managing relationships with the different agents in the distribution channel contribute to enhance tourism businesses' access to their target, a need intensified by the intangibility of the product bid and the consequent trust requirement by the customer.

Under the stakeholder marketing approach, it is proposed the management of every agent in the channel, being this approach an especially useful tool for managing interest and trust relationships between both of them. To analyse this phenomenon and to illustrate the utility of the proposed approach, under the case study methodology, this research will be focused on the firm Bookingfax, which makes a profit of its relational capital as the base of its business model.
\end{abstract}

\section{Keywords}

value chain, marketing, stakeholder, touroperator, relationships, travel agencies

\section{INTRODUCCIÓN}

El uso de las tecnologías de la comunicación y de la información por parte de los consumidores se ha ido transformando en la última década, destacando la centralidad de las redes sociales en este cambio. Los consumidores usan cada vez más sus terminales móviles para contratar servicios turísticos y las redes sociales se han convertido en una fuente de información fundamental para la toma de decisiones y la planificación en materia de viajes. Así, las relaciones de las empresas del sector turístico deben aprovechar el potencial de las redes sociales para acceder al mercado, usando para ello un enfoque relacional, como es el marketing de

\footnotetext{
${ }^{1}$ Doctor en Administración y Dirección de Empresas
} 
stakeholders, un modelo de negocio que se ilustrará a través de un estudio de caso enfocado en la empresa Bookingfax, cuyo negocio siempre ha capitalizado sus relaciones en el sector conectando a turoperadores y usuarios finales y simultáneamente ha sabido transformar su actividad conforme a las exigencias que los consumidores establecen con su comportamiento de compra de servicios turísticos y con el uso que éstos realizan de sus redes sociales en relación a esta actividad.

\section{EL MARKETING Y EL PARADIGMA RELACIONAL}

El marketing se define actualmente como "la actividad, el conjunto de instituciones y procesos para crear, comunicar, distribuir e intercambiar ofertas de valor para los clientes, los socios y la sociedad en general" (American Marketing Association, AMA, 2013). Esta definición recoge el enfoque tradicional de marketing basado en el proceso descrito en el marketing-mix (Borden, 1964). Sin embargo, este enfoque se basa en una visión interna de la empresa, centrada en el proceso de gestión de marketing, en contraposición a la actual orientación al cliente, siendo ésta una de las principales argumentaciones a favor del paradigma relacional de marketing (Grönroos, 1994; Prahalad y Ramaswamy, 2004; Svensson, 2001).

El paradigma relacional de marketing sostiene que el elemento principal de marketing, o núcleo conceptual, y sobre el que se sostiene la actividad de la empresa, es la gestión de las relaciones y no el intercambio, como se ha venido aceptando en las últimas décadas. Inicialmente, el marketing relacional surge como un medio para incrementar la fidelidad del consumidor mediante el conocimiento exhaustivo del mismo (Arndt, 1979; Berry, 1983), sin embargo pronto empieza a reclamarse el papel central del mismo por la falta de orientación al cliente del modelo de gestión imperante (Grönroos, 1994) y a reconocerse la influencia de otros actores en el resultado de la empresa tanto en la definición que daba la propia AMA (2004) como por parte de la Academia (AMA, 2008; Grönroos, 1991; Gundlach y Wilkie, 2009 y 2010; Hult et al, 2011; Dibb et al, 2014).

Lamberti y Noci (2010) recogen esta evolución del enfoque relacional de las empresas, entendiendo que su gestión va desde el manejo de una simple base de datos, a la interacción colaborativa en un enfoque uno a uno, para terminar en una visión de red en la que las relaciones son muchos a muchos, siendo necesarias todas esas relaciones de colaboración para que la red optimice su rendimiento.

Profundizando en esta visión de la empresa, Kotler, Jain y Maesincee (2004) señalan que para que una empresa sobreviva en el contexto económico actual es necesario no sólo crear valor para el cliente y gestionar las propias competencias esenciales, sino también la gestión de las redes de colaboración de la empresa.

Bajo este paradigma surge el concepto de marketing de stakeholders, el cual se define como "las actividades dentro de un sistema de instituciones sociales y los procesos para facilitar y mantener el valor a través de relaciones de intercambio con múltiples grupos de interés" (Hult et al, 2011). En definitiva, supone el reconocimiento del entramado de relaciones de intercambio de valor en el que la empresa se ve inmersa para desarrollar su actividad y alcanzar el objetivo de lograr el intercambio con sus clientes.

Siguiendo esta concepción de las relaciones empresariales, la empresa ocupará una posición en el mercado o sector tan fuerte como el valor que sea capaz de generar con sus relaciones, por lo que deberá conocer las demandas de sus distintos stakeholders o grupos de interés, siendo necesaria una comunicación fluida, regular y bidireccional con cada uno de ellos, priorizando la satisfacción de dichas demandas en función de su importancia en la consecución del objetivo de lograr intercambios de valor con el cliente (Frow y Payne, 2011; Gessa y Jiménez, 2011; Hult et al, 2011; Payne y Frow, 2005; Payne et al, 2005)

\section{LA APLICACIÓN DE LAS REDES SOCIALES Y EL MARKETING DE STAKEHOLDERS A LOS SERVICIOS TURÍSTICOS}

Dado que los servicios turísticos son de naturaleza intangible y además se prestan en un lugar y por personas distintas al punto de venta, el éxito de la oferta turística tanto global como de cualquiera de sus componentes descansa en gran medida en la confianza generada en el cliente final.

De la misma manera, la satisfacción del consumidor turístico vendrá dada por la experiencia total que viva tanto en el tránsito hacia el destino turístico y de vuelta de él como en el propio destino (Baggio y Cooper, 2010; Volo, 2013), por lo que la cooperación de los distintos agentes que toman parte en esa experiencia, sean entidades públicas gestoras del destino o empresas turísticas deben relacionarse y colaborar para lograr ofrecer al turista una experiencia lo más satisfactoria posible, incrementando las posibilidades tanto de una primera compra como de recomendación a familiares y amigos.

Esta visión de la actividad del sector turístico hace especialmente necesaria la gestión de las relaciones y la consideración de que otros entes distintos al cliente deben ser tenidos en cuenta como demanda de la empresa si bien no se va a producir necesariamente una transacción comercial con ellos, sino un intercambio de valor. Como puede en la definición de marketing de stakeholders, el objeto de esta visión de la empresa coincide con la 
propia naturaleza de la actividad turística y su gestión, por lo que su aplicación a las empresas del sector es especialmente recomendable. Respecto a la forma de aplicarlo, pueden distinguirse varias tareas clave (Payne y Frow, 2005; Payne et al, 2005; Frow y Payne, 2011):

- Identificar los grupos de interés relevantes: en este caso, aquellos agentes del canal que pueden contribuir a generar la confianza necesaria en el cliente o incluso a alcanzarlo. Dado que las relaciones son dinámicas, el número lo la importancia de los stakeholders pueden variar con el paso del tiempo.

- Comunicarse con los distintos stakeholders para poder estudiar cuáles son sus demandas específicas o qué esperan de la empresa.

- Priorizar la satisfacción de dichas demandas en función de su importancia en relación a su contribución a lograr los objetivos marcados siempre que éstas sean razonables.

- Elaborar una estrategia de relaciones adecuada usando para ello la información obtenida en los tres pasos anteriores.

La aplicación de este procedimiento en las empresas del sector turístico las beneficia en una doble vía:

- Porque mejore la percepción que el cliente tiene de su experiencia turística global, dado que la colaboración con otros agentes de un mismo destino puede producir este efecto.

- Porque puede obtener acceso y mejorar la confianza de clientes con los que no tiene una vía de comunicación directa o ésta es muy costosa.

Por otra parte, y en su aplicación directa a las empresas y operadores turísticos y cómo éstos utilizan las redes sociales y la web 2.0 y sus posibilidades de interacción con el cliente, el modelo relacional descrito es una herramienta por aprovechar, pues estos agentes no han utilizado hasta ahora ninguna de las principales herramientas de manera efectiva (Minghetti y Buhalis, 2010). Las tecnologías de la información facilitan la comunicación con los distintos grupos de interés, pero simultáneamente hacen su gestión más compleja por el número de ellos que pueden alcanzarse en la red y sus tipologías, si bien son una necesidad de gestión actualmente (Hvass y Munar, 2012).

Sin embargo, el crecimiento de uso que están teniendo redes como Facebook o Twitter aplicados a la búsqueda de contenidos turísticos, en algunos casos con tasas del orden del $1000 \%$, hacen aún más urgente la adopción de estas herramientas relacionales por parte del sector, teniendo en cuenta el tipo de contenidos y el formato que los consumidores van a demandar (Milano et al, 2011), teniendo especial preferencia por los visuales y aquellos que les aportan valor de uso, por encima de la narrativa o los formatos más clásicos (Munar y Jacobsen, 2014).

\section{METODOLOGÍA}

La estrategia de investigación a seguir en este trabajo se basa en la metodología del estudio de casos, que se define como "una investigación empírica que investiga un fenómeno contemporáneo en su contexto real, donde los límites entre el fenómeno y el contexto no se muestran de forma precisa, y en el que múltiples fuentes de evidencia son usadas" (Yin, 1989). Esta metodología constituye por tanto una herramienta que permite, entre otros cometidos (Chetty, 1996):

Estudiar temas de investigación en el que las teorías existentes son inadecuadas.

Estudiar fenómenos desde múltiples perspectivas y no desde la influencia de una sola variable.

Explorar en forma más profunda y obtener un conocimiento más amplio sobre cada fenómeno.

Aunque el marketing relacional como concepto ha tenido una larga evolución desde principios de los años 80 del siglo pasado, su concepción como enfoque integral de gestión de la empresa es reciente, por lo que se puede considerar como en fase exploratoria, estando sujeto a debate y a un mayor desarrollo teórico. Igualmente, la complejidad y la intervención de diversos sujetos en una red de relaciones sociales o empresariales hacen necesario un enfoque lo suficientemente flexible como para poder apreciar cuestiones emergentes en el desarrollo de la investigación.

Sin embargo, esta flexibilidad no debe ser interpretada como falta de rigor y consistencia de los datos utilizados, por lo que en esta investigación se sigue el esquema propuesto por Yin (2009) y que Villarreal y Landeta (2010) sistematizaron en mayor profundidad, garantizando la triangulación teórica y de fuentes y por lo tanto la fiabilidad de las conclusiones extraídas.

El estudio en este caso se basa en varias fuentes de información, como son una entrevista personal al CEO de Bookingfax S.L., Antonio Mariscal, los datos ofrecidos a través de la web de la compañía, y un extracto de los estados de tráfico de datos de la compañía, así como la contrastación de las afirmaciones y datos ofrecidos a 
través de terceros, como pueden ser los perfiles de redes sociales (Facebook y Twitter) de agencias de viajes que utilizan la API de Bookingfax S.L.

Así, se puede resumir el enfoque metodológico, siguiendo la metodología de estudio de casos, como un estudio de caso único (con varios agentes) y en el que se estudian las relaciones existentes entre ellos desde un enfoque holístico (ver tabla 4.1).

Tabla 1. Ficha técnica del estudio de casos Bookingfax

Fuente: Elaboración propia a partir de Villarreal y Landeta (2010)

\begin{tabular}{|c|c|}
\hline $\begin{array}{l}\text { Propósito de la } \\
\text { investigación }\end{array}$ & $\begin{array}{l}\text { Analizar la validez del paradigma relacional a través del marketing de } \\
\text { stakeholders como un medio para la gestión del valor de la empresa. }\end{array}$ \\
\hline Metodología & $\begin{array}{l}\text { Estudio de casos contemporáneo simple de carácter holístico. Naturaleza } \\
\text { exploratoria, descriptiva y explicativa. }\end{array}$ \\
\hline Unidad de análisis & Bookingfax S.L. y su red de actuación. \\
\hline Tipo de muestra & $\begin{array}{l}\text { Intencional, motivada por el acceso a los datos y la naturaleza de servicio de } \\
\text { la empresa. }\end{array}$ \\
\hline Muestra & Bookingfax S.L. \\
\hline $\begin{array}{l}\text { Métodos de recogida } \\
\text { de evidencia }\end{array}$ & $\begin{array}{ll}\text { - } & \text { Entrevista personal grabada en audio. } \\
\text { - } & \text { Notas del entrevistador. } \\
\text { - } & \text { Información publicada en web corporativa. } \\
\text { - } & \text { Capturas del sistema de administración de la empresa. } \\
\text { - } & \text { Perfiles de Facebook y Twitter de Agencias usuarias de servicios de } \\
& \text { Bookingfax. }\end{array}$ \\
\hline Informadores clave & Antonio Mariscal, CEO de Bookingfax S.L. \\
\hline $\begin{array}{l}\text { Métodos de análisis de } \\
\text { evidencia }\end{array}$ & $\begin{array}{cl}\text { Fundamentalmente cualitativos: } \\
\text { - } & \text { Indentificación de agentes y sus relaciones. } \\
\text { - } & \text { Contrastación con la teoría propuesta. } \\
\text { - } & \text { Contraste con otras teorías. } \\
\text { - } & \text { Identificación de factores clave y relaciones causales. } \\
\text { - } & \text { Análisis de relaciones críticas. }\end{array}$ \\
\hline Enfoque científico & Inductivo, basado en la lógica de su replicabilidad (generalización analítica). \\
\hline $\begin{array}{l}\text { Evaluación del rigor y } \\
\text { calidad metodológica }\end{array}$ & Asegurada mediante triangulación de datos y teórica. \\
\hline Fecha de realización & Abril de 2015. \\
\hline
\end{tabular}

Una vez recopilada la información necesaria, ésta fue analizada teniendo como enfoque general la cadena de valor entre los tour operadores u oficinas de turismo y los consumidores finales, centrando la atención sobre las relaciones que se dan en el canal.

\section{EL CASO BOOKINGFAX}

La empresa Bookingfax S.L., con sede en Jerez de la Frontera (Cádiz, España), nace en 2002 como intermediario entre mayoristas y agencias de viajes, optimizando el envío de ofertas de paquetes turísticos mediante fax a éstas últimas. La evolución de las telecomunicaciones y en particular del uso que los consumidores hacen de éstas y cómo acceden a Internet y a las redes sociales cuando buscan información o quieren contratar productos turísticos han llevado a retornar a las agencias de viajes parte del mercado que perdieron con el nacimiento de las agencias online.

Paralelamente Bookingfax S.L. ha ido modificando la naturaleza de sus servicios y ha pasado de distribuir ofertas por fax a colocarlas en los perfiles de redes sociales de sus usuarios mediante una aplicación propia. Sin embargo, la base del negocio sigue siendo la misma: Bookingfax S.L. capitaliza la confianza que las agencias depositan en sus servicios gratuitos mediante el cobro de lanzamiento de ofertas a los mayoristas (turoperadores) o a agencias gestoras de destinos turísticos.

Bookingfax S.L. divide su cartera de servicios en 2 grandes grupos: agencias (y grupos de agencias) y mayoristas, proveedores y oficinas de turismo. A los primeros les ofrece contenidos para sus perfiles en redes sociales, así como la comunicación de ofertas de paquetes en tiempo real todo ello de manera gratuita. A los mayoristas, proveedores y oficinas de turismo les cobra por introducir sus ofertas y contenidos en los perfiles de las agencias. Los generadores de oferta están muy lejos del consumidor de productos turísticos ya que no tienen acceso a ellos y dependen de las agencias para poder contactar con ellos. El consumidor conoce a la agencia y las personas responsables de ella, lo que le ofrece la confianza necesaria para adquirir un producto intangible como es un paquete vacacional. Sin embargo, en la mayoría de ocasiones éste desconoce si el proveedor del mismo es Pullmantur, Thomas Cook, o Catai, marcas que no ofrecen ningún estímulo para él. En conclusión, los proveedores necesitan acceder al cliente y las agencias necesitan contenidos y ofertas con las que atraer al cliente a su punto de venta. Se necesitan mutuamente, pero lanzar una oferta de manera eficaz a la gran 
diversidad de agencias existentes y que éstas hagan llegar la oferta al consumidor final es un proceso costoso y no garantizado para el turoperador o la oficina de turismo y es en este punto en el que Bookingfax S.L. ofrece valor a toda la cadena, ya que reduce costes para el turoperador, que prefiere externalizar esta comunicación, y optimiza la utilidad comercial de las redes sociales para la agencia, que ve como sus clientes entran en la oficina empujados por la oferta que han visto vía Facebook o Twitter.

En definitiva, podemos identificar a 6 agentes participantes en este mercado:

Tour operadores y mayoristas.

Oficinas de turismo.

Bookingfax S.L.

Redes Sociales.

Agencias de Viajes.

Consumidor final.

Todos ellos establecen relaciones de mutuo beneficio entre sí de manera directa o indirecta, por lo que la gestión de dichas relaciones determinará la necesidad de cada agente en el canal (Ver Gráfico 5.1.).

En primer lugar es necesario poner de manifiesto que dentro del sector turístico, las ofertas que se lanzan al consumidor final han pasado por un proceso en el que el agente tradicional perdía peso como canal de venta a favor de portales o agencias web, sin embargo, la falta de confianza del cliente para productos turísticos en los que dicho factor es clave hacen que éstas sigan siendo el principal canal para esa categoría de producto en la que figuran los grandes circuitos, los cruceros y ofertas similares. En este contexto la necesidad del agente es atraer al cliente al punto de venta, para lo cual necesita contenidos atractivos vía redes sociales, para obtener la atención de su audiencia cercana. Bookingfax S.L. aporta esa función a la agencia de viajes de manera gratuita, permitiéndole filtrar y configurar el tipo de ofertas que van a ser publicadas de manera automática en su perfil de Facebook o Twitter. Bookingfax S.L. a cambio obtiene una gran masa de agencias de viaje que le permite exigir un precio a quien desee colocar una oferta en esta red.

Por otra parte, Bookingfax S.L. ofrece a los tour operadores y a las oficinas de turismo alcanzar al cliente final a un menor coste, obteniendo ingresos por ello. Es decir, el verdadero cliente de Bookingfax S.L. no son los consumidores turísticos, sino las empresas o instituciones que desean llegar a éstos.

Así, se aprecia como las relaciones entre los distintos agentes dan sentido a la actividad de Bookingfax S.L., y como la empresa posibilita mediante sus relaciones con éstos la correcta actividad de los mismos. Dicho en otras palabras, toda la actividad empresarial desarrollada por los distintos miembros del canal se basa en las relaciones que desarrollan con los demás agentes.

Por último, el enfoque predominante en marketing, basado en el marketing-mix, considera que la planificación de las actividades de marketing debe realizarse en torno a 4 grandes variables: producto, precio, comunicación y distribución. En este caso se pone de manifiesto que los turoperadores, si bien son capaces de generar ofertas (producto) atractivas en precio y contenido, no consiguen alcanzar al consumidor final, por coste o por falta de acceso, y la distribución de sus ofertas no es eficiente siguiendo el canal tradicional, ya que los clientes necesitan ser atraídos al punto de venta y el envío de las ofertas a las agencias por los medios tradicionales no garantiza que lleguen al cliente final.

\subsection{Relaciones entre los distintos agentes del canal de distribución de ofertas turísticas.}

La actividad de Bookingfax aporta valor en una doble vía:

A sus clientes de pago (turoperadores y oficinas de turismo) mediante la distribución eficiente de ofertas. Éstos pueden llegar a un mayor número de usuarios finales, y no agencias, tal y como ponen a disposición de Bookingfax una oferta, ya que esta empresa puede publicarlas en tiempo real en el muro de las distintas agencias cliente. Bookingfax aporta por lo tanto un mayor número de receptores reales (que la oferta llegue a la agencia no implica que el cliente tenga conocimiento de ella) al turoperador y también una mayor rapidez y menor coste por impacto, por lo que aumenta la eficiencia en la distribución de ofertas.

A los clientes gratuitos les aporta tráfico físico en las agencias. La colocación de ofertas atractivas desde el punto de vista económico, del contenido y de la flexibilidad visual que aportan las redes sociales capta la atención de los seguidores de las agencias en dichas redes (con mayor efectividad que los medios tradicionales) y despierta su interés, dirigiéndolos a la agencia para ampliar información y eventualmente contratar el servicio. Además, las agencias de viaje pueden olvidar la tarea de mantener y actualizar sus perfiles de redes sociales, una tarea que no suele entrar en las prioridades de dichas empresas y que en muchos casos resulta complicada desde el punto de vista técnico o de 
generación de contenidos, a pesar de que consideren que esta herramienta colabore en la obtención de un mayor volumen de ventas (Bookingfax S.L., 2015).

- A pesar de que el consumidor turístico no es cliente de Bookingfax ni mantiene una relación directa con la empresa, la generación de contenidos de manera automática en los perfiles de las agencias hace que éste disponga de información de interés en la planificación de viajes o en el conocimiento de nuevos destinos, siendo esta última utilidad del consumidor el objetivo de las oficinas de turismo que contratan a Bookingfax para la distribución de material promocional.

Por esta razón, el papel de Bookingfax, cuya actividad es esencialmente relacional, conecta a los 3 agentes mencionados, aportando eficiencia en la distribución con un elemento, las relaciones, que no se contempla de manera explícita en el enfoque de marketing prevalente, de ahí la necesidad de estudiar, perfeccionar y divulgar un concepto como el de marketing de stakeholders y valorar la viabilidad de un paradigma relacional en marketing.

Imagen 1. Fuente: elaboración propia.

\section{Turoperador}

\section{Ingresos}

Acceso inmediato al consumidor

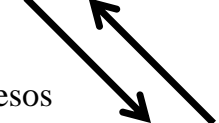

\section{Masa crítica de}

relaciones con el consumidor final

Ingresos

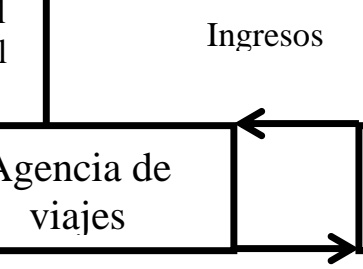

Consumidor turístico
Ingresos

Acceso al consumidor final y reconocimiento de marca
Atracción gratuita de clientes al punto de venta
Confianza y garantía en la compra de productos turísticos complejos

Oficina de

turismo

\section{CONCLUSIONES}

Teniendo en cuenta tanto las prescripciones de los trabajos centrados en la gestión de las relaciones empresariales (Prahalad y Ramaswamy, 2004; Vargo y Lusch, 2004; Payne y Frow, 2005; Payne et al, 2005; Frow y Payne, 2011) como el análisis de los datos obtenidos en la investigación empírica llevada a cabo en Bookingfax S.L. se puede llegar a una primera conclusión consistente en la validez de la gestión de las relaciones como método esencial para crear valor.

Además, tal y como indica Antonio Mariscal, CEO de Bookingfax S.L., el paso de un modelo de negocio basado en la gestión de ofertas por fax que constituía el planteamiento inicial de la empresa al modelo actual, basado únicamente en la gestión de relaciones a través de medios digitales, descansa en gran medida en la confianza generada con las relaciones construidas y mantenidas desde la etapa anterior, que han sido capitalizadas en un proceso de adaptación tecnológica, que hubiera sido o bien imposible o bien extremadamente costoso y arriesgado de haber partido de cero contando únicamente con el componente tecnológico y despreciando el capital relacional de la empresa.

Por otra parte, la supervivencia de la agencia de viajes tradicional frente al auge de la distribución de productos turísticos online se debe precisamente a las relaciones de confianza que éstas han construido y mantenido con sus clientes, los cuáles no encuentran la misma garantía en la contratación online, especialmente para productos de especialidad como un circuito, viajes de larga distancia que implican un alto coste, o cruceros, en los que la necesidad de información es mayor y la autonomía del viajero se reduce. 
Más allá de las relaciones que las agencias mantienen con sus clientes, y por consiguiente de la capacidad de segmentación que éstas obtienen de dicho conocimiento, Bookingfax S.L. y las agencias de viajes, a través de sus perfiles de redes sociales, están cogenerando o cocreando valor para sus respectivos clientes (Prahalad y Ramaswamy, 2004; Vargo y Lusch, 2004), ya que sus respectivos clientes generan valor para el otro. Es decir, el cliente de la agencia es al que desea alcanzar el tour operador, y a la vez el cliente de la agencia desea acceder a contenidos y ofertas de viajes de manera fácil y personalizada.

La importancia de las relaciones de cogeneración/cocreación de valor se pone de manifiesto igualmente ante la facilidad con la que puede producirse una confusión en la distinción de quién es el cliente final en esta cadena de valor, ya que puede ser recorrida en ambos sentidos, y tanto el turista como el turoperador pueden ser considerados bajo dicho rol. Es la actividad conjunta de consumidores, agentes y Bookingfax lo que resulta atractivo al turoperador, por ejemplo, y no la actividad de uno de ellos de manera aislada. Además, el turoperador genera parte del servicio que contrata, al ser éste el que diseña la oferta a comunicar y los medios audiovisuales a emplear.

Centrando la atención en el modelo teórico propuesto, el marketing de stakeholders o marketing de grupos de interés, y en relación al mercado turístico, el uso de las redes sociales resulta una herramienta básica y barata para alcanzar al cliente y a cada uno de los grupos de referencia, siendo refrendada esta afirmación por las propias agencias, las cuales consideran en un $57 \%$ que las redes sociales han contribuido a mejorar sus ventas (Bookingfax S.L. 2015).

Si se analizan las relaciones que se dan en el canal de distribución de las ofertas turísticas, el turoperador no cede la propiedad ni el riesgo de las ofertas que confecciona a ninguno de los miembros del canal, por lo que la "propiedad" del viaje pasa directamente de éste al consumidor, sin embargo, la colaboración de los distintos agentes es esencial para lograr alcanzar este intercambio. En otras palabras, es posible vender ofertas de viaje gracias a que existen relaciones de confianza y cooperación de mutuo beneficio entre los distintos miembros del canal, de manera que la satisfacción de estos intereses recíprocos mantiene en funcionamiento la red.

Finalmente, se puede concluir a la vista del caso estudiado que la gestión de las relaciones del canal, en este caso bajo la iniciativa de Bookingfax S.L. ha aportado a los distintos miembros los siguientes beneficios:

Optimización de los costes de alcance al cliente.

Revitalización del canal tradicional y del papel de los agentes de viajes y de los turoperadores frente a la desintegración del producto turístico con la difusión de internet en las últimas dos décadas.

Atracción de clientes al punto de venta físico.

- Reducción a casi cero del time-to-market en la distribución de ofertas de turoperadores.

Por todas estas razones, se considera que la adopción de un enfoque relacional basado en la atención a los distintos grupos de interés no sólo va a beneficiar a las empresas o agentes que lo adopten, sino que va a proporcionar una mayor eficiencia y valor a la cadena en la que desarrollen su actividad.

\section{BIBLIOGRAFÍA}

AMERICAN MARKETING ASSOCIATION (AMA) (2008): The American Marketing Association Releases New for Marketing. Disponible en https://archive.ama.org/archive/AboutAMA/Documents/American\%20Marketing\%20Association\%20Rele ases\%20New\%20Definition\%20for\%20Marketing.pdf Consultado en Abril de 2015.

AMERICAN MARKETING ASSOCIATION (AMA) (2013): Definition of Marketing. Disponible en https://www.ama.org/AboutAMA/Pages/Definition-of-Marketing.aspx Consultado en Abril de 2015.

ARNDT, J. (1978). How Broad Should the Marketing Concept Be? Journal of Marketing 42, 101-103.

BAGGIO, R. y COOPER, C. (2010): "Knowledge transfer in a tourism destination: the effects of a network structure." The Service Industries Journal: 30: 10: 1757-1771.

BERRY, L. L. (1983). Relationship marketing. In L. L. Barry, G. L. Shostack, \& G. D. Upah (Eds.), Emerging perspectives on services marketing (pp. 25 - 28). UT: American Marketing Association.

BOOKINGFAX, S.L. (2015): Redes Sociales y Agencias de Viajes. Estadísticas rápidas. Jerez de la Frontera, Cádiz, Febrero de 2015.

BORDEN, N. H. (1964). The concept of the marketing mix. Journal of advertising research, 4(2), 2-7.

DIBB, S., SIMÕES, C. y WENSLEY, R. (2014): Establishing the scope of marketing practice: insights from practitioners. European Journal of Marketing. 48 (1-2), 380-404.

FROW, P. y PAYNE, A (2011). A stakeholder perspective of the value proposition concept. European Journal of 
Marketing, 45(1/2), 223-240.

GESSA, A. y JIMÉNEZ, M.A. (2011): La gestión de las relaciones con los grupos de interés. Un análisis exploratorio de casos en el sector hotelero español. Estudios y perspectivas en turismo. 20, 753- 771.

GRÖNROOS, C. (1991). The marketing strategy continuum: towards a marketing concept for the 1990s. Management decision, 29(1).

GRÖNROOS, C. (1994). From marketing mix to relationship marketing: towards a paradigm shift in marketing. Management decision, 32 (2), 4-20.

GUNDLACH, G. y WILKIE, W. (2009). The American Marketing Association's New Definition of Marketing: Perspective and Commentary on the 2007 Revision. Journal of Public Policy \& Marketing 26 (2), 259.

GUNDLACH, G. y WILKIE, W. (2010). Stakeholder Marketing: Why "Stakeholder" Was Omitted from the American Marketing Association's Official 2007 Definition of Marketing and Why the Future Is Bright for Stakeholder Marketing. Journal of Public Policy \& Marketing 29 (1), 89.

HULT, G.T.M., MENA, J.A, FERRELL, O.C. y FERRELL, L. (2011). Stakeholder marketing: a definition and conceptual framework. Academy of Marketing Science Review 1 (1), 44-65.

HVASS, K.A. y MUNAR, A.M. (2012): The takeoff of social media in tourism. Journal of Vacation Marketing. 18(2), 93-103.

LAMBERTI, L. y NOCI, G. (2010): Marketing strategy and marketing performance measurement system: Exploring the relationship. European Management Journal. 28, 139- 152.

MILANO, R.; BAGGIO, R. y PIATTELLI, R. (2011): The effects of online social media on tourism websites. $18^{\text {th }}$ International Conference on Information Technology and Travel \& Tourism. Inssbruck, Austria.

MINGHETTI, V. Y BUHALIS, D. (2010): Digital Divide in Tourism. Journal of Travel Research 49, 267-281.

MUNAR, A.M. y JACOBSEN, J.K.S. (2014): Motivations for sharing tourism experiences through social media. Tourism Management 43, 46-54.

PAYNE, A.F., BALLANTYNE, D. y CHRISTOPHER, M. (2005): A stakeholder approach to relationship marketing strategy. The development and use of the "six markets" model. European Journal of Marketing. 39 (7-8), 855- 871 .

PAYNE, A.F. y FROW, P. (2005): A Strategic Framework for Customer Relationship Management. Journal of Marketing. 69, 167-176.

PRAHALAD, C.K. y RAMASWAMY, V. (2004). Co-Creation Experiences: The Next Practice in Value Creation. Journal of Interactive Marketing, 18 (3), 5- 14.

SVENSSON, G. (2001). Re-evaluating the marketing concept. European Business Review, 13 (2), 95-100.

VARGO, S.L. y LUSCH, R.F. (2004). Evolving to a New Dominant Logic for Marketing. Journal of Marketing, 68 (1), 1-17.

VILLAREAL, O. y LANDETA, J. (2010): "El estudio de casos como metodología de investigación científica en dirección y economía de la empresa. Una aplicación a la internacionalización." Investigaciones Europeas de Dirección y Economía de la Empresa Vol. 16 nำ pp.31-52.

VOLO, S. (2013): "Conceptualizing Experience: A Tourist Based Approach". En Marketing of Tourism Experiences, Editado por Scott, N.; Laws, E. y Boksberger, P. en Ed. Routledge. pp. 13-28.

YIN, R.K. (2009): "Case Study Research: Design and Methods." SAGE Publications, Thousand Oaks, CA, Estados Unidos. 